\title{
A case report of megalencephalic leukoencephalopathy with subcortical cysts
}

Keywords: macrocephaly, MRI, neuroregression, dysarthria, leukodystrophy

Abbreviations: MRI, magnetic resonance imaging; MRS, magnetic resonance spectroscopy; NAA, N-acetyl aspartate

\section{Introduction}

Megalencephalic leukoencephalopathy with subcortical cysts is a rare degenerative disease first described by Vander knap. ${ }^{1}$ It is clinically characterized by early onset enlarged head (macrocephaly), mild motor developmental delay, and seizures. Later, patients may develop gradual onset of ataxia and pyramidal features and mental deterioration. ${ }^{2}$ The combination of clinical findings and MRI features is essential for the diagnosis. The disease has autosomal recessive inheritance and the gene locus is mapped to MLC1 at chromosome $22 \mathrm{q} .^{3}$

\section{Case details}

24years old male is 1 st born to consanguinous parentage from non agarwal community. Patient has normal perinatal history and normal development up to 13years of age except macrocephaly. From 13 years of age neuroregression was noted in the form of decreased attained scholastic performance, loss of communication skills, slowness of activities and development of seizures. Since 19years age there was gradually progressive asymmetric spastic quadriparesis with associated spastic dysarthria. Significant family history was noted with similar history of neruoregression was in younger sibling. On examination macrocephaly was seen, patient was conscious with
Volume 9 Issue 5 - 2019

\section{Sampath kumarNS, Sangamithra G, Shyam sundar M, Surya Teja P \\ Department of Neurology, Narayana Medical college \& Hospital, Nellore, AP}

\section{Correspondence: Sangamithra G, DM Neurology,Assistant professor, Department of Neurology, Narayana Medical College, India, Tel 98495568I5,}

Email drsangamithrag@narayanamedicalcollege.com

Received: June 18, 2019 | Published:September 12, 2019

decreased attention, left UMN facial palsy with spastic dysarthria noted and spastic quadriparesis with gross incoordination was also observed.

\section{Investigations}

\section{a. MRI}

MRI Brain axial T2w images showed multiple subcortical cysts in the bilateral temporoparietal regions and diffuse hyperintense white matter changes (Figure 1).

\section{b. Genetic analysis}

DNA sample analysis for mutaion on MLC1 gene showed heterogeneous mutation in exon 2 confirming the diagnosis of MLC.
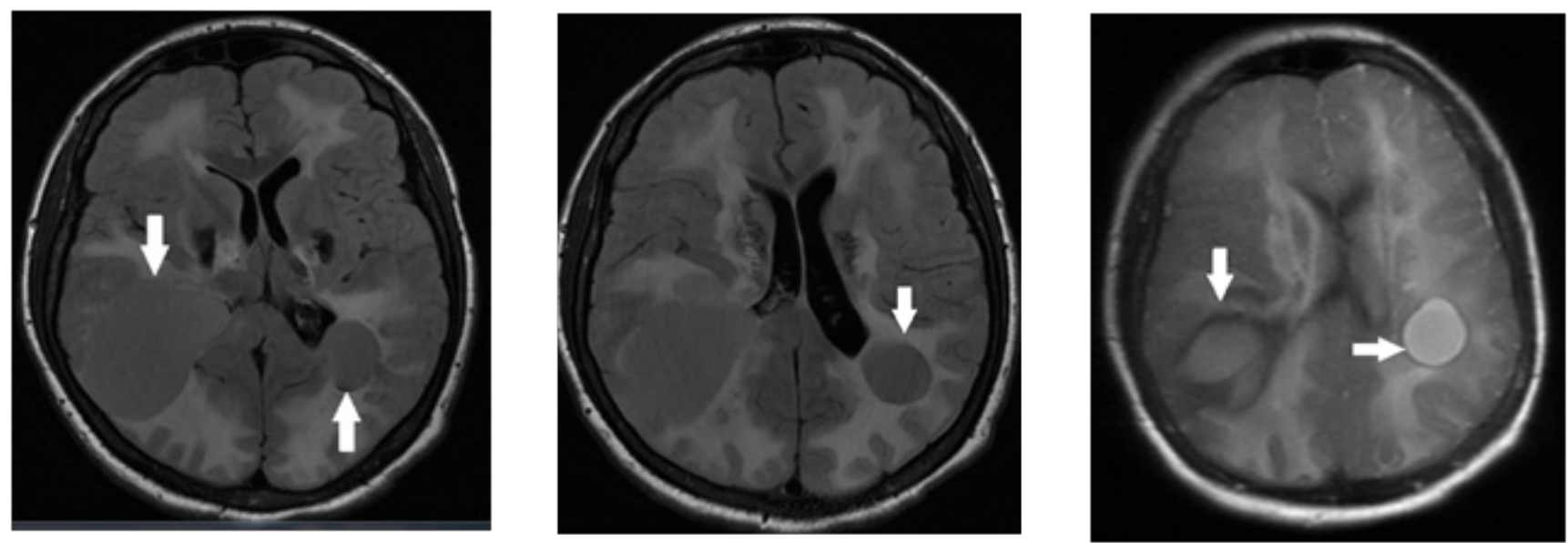

Figure I MRI Brain axialT2w images showed multiple subcortical cysts in the bilateral temporoparietal regions and diffuse hyperintense white matter changes.

\section{Discussion}

MLC is the most common leukodystrophy with megalencephaly observed in India. It is usually observed in the agarwal community. ${ }^{4}$ The disease has high incidence in populations in which consanguinity is common. MLC is an autosomal recessive disorder due to mutations in the MLC1 gene which has locus in chromosome 22q. Severity of phenotype does not correlate with the specific mutations found. ${ }^{5}$ The diagnosis of MLC can be made with typical clinical features and characteristic abnormalities on cranial MRI. ${ }^{6}$

Leukoencephalopathy can present as abnormal muscle tone (spasticity), difficulty coordinating movements (ataxia), cysts in 
the brain (subcortical cysts), abnormal muscle posturing (dystonia), swallowing difficulties, mild to moderate intellectual disabilities, speech difficulties, seizures, and difficulties walking.

Clinical findings usually consist of macrocephaly at birth or in $1^{\text {st }}$ year of life with normal or mild developmental delay and slow deterioration of motor functions with cerebellar ataxia. Mild spasticity usually starts in early childhood or later. The majority of affected children become wheelchair dependent in their teens. Speech can become increasingly dysarthric; dysphagia may develop in late stages. Although there is no specific treatment or cure for MLC, there are ways to manage the symptoms, such as use of antiepileptic drugs, physical therapy, and speech therapy.

\section{MRI criteria}

MRI of the brain is diagnostic. Cerebral hemispheric white matter is diffusely abnormal and mildly swollen. Subcortical cysts are almost invariable in the anterior temporal region and often in the frontoparietal region. Central white matter structures, including the corpus callosum, internal capsule, and brain stem, are better preserved. The magnetic resonance spectroscopy [MRS] findings in this disorder include mild to moderate decreases in the $\mathrm{N}$-acetyl aspartate [NAA] to choline and choline to creatine ratios. ${ }^{7}$

\section{Differential diagnosis}

The combination of megalencephaly and leukoencephalopathy along with the typical MRI findings are seen in a limited number of disorders such as MLC, Canavan disease, Alexander disease, infantile-onset GM2 gangliosidosis, glutaric aciduria type 1, and merosin-deficient congenital muscular dystrophy. In Canavan disease, NAA is elevated in urine and blood and a deficiency of the enzyme aspartoacylase can be demonstrated in cultured fibroblasts. 8 In Alexander disease cystic degeneration may occur but the location of the cysts is different: The deep frontal white matter is mainly affected. ${ }^{9}$ MRI in infantile GM2 gangliosidosis is characterized by prominent involvement of the basal ganglia and thalami in addition to the white matter abnormalities. ${ }^{10}$ In merosin deficient congenital muscular dystrophy, white matter involvement resembles that observed in MLC, but the typical subcortical cysts are generally lacking. Prominent weakness and hypotonia are commonly seen in patients with merosin deficient congenital muscular dystrophy which are rare findings in MLC. ${ }^{11}$

\section{Conclusion}

MLC should be considered in the differential diagnosis of children with megalencephaly and leukoencephalopathy. One should suspect and carryout genetic tests to confirm the diagnosis because it has a remarkably slow course of deterioration in neurologic function and early rehabilitation may prolong ambulatory life.

\section{Acknowledgments}

None.

\section{Conflict of interest}

The authors declare that there is no conflict of interest.

\section{Funding}

None.

\section{References}

1. Van Der Knaap MS, Barth PG, Stroink H, et al. Leukoencephalopathy with swelling and a discrepantly mild clinical course in eight children. Ann Neurol. 1995;37:324-334.

2. Sethi PK, Sethi NK. Megalencephalic leukoencephalopathy with subcortical cysts in all three siblings of a non-Aggarwal Indian family. Ann Indian Acad Neurol. 2012;15(3):214-217.

3. Koussa S, Roukoz H, Rizk T, et al. Megalencephalic leucoencephalopathy with subcortical cysts: A study of a Lebanese family and a review of the literature. Rev Neurol (Paris). 2005;161:183-191.

4. Singhal BS, Gursahani RD, Udani VP, et al. Megalencephalic leukodystrophy in an Asian Indian ethnic group. Pediatr Neurol. 1996;14:291-296.

5. Leegwater PA, Yuan BQ, van der Steen J, et al. Mutations of MLC1 (KIAA0027), encoding a putative membrane protein, cause megalencephalic leukoencephalopathy with subcortical cysts. Am J Hum Genet. 2001;68:831-838.

6. Topçu M, Saatci I, Topcuoglu MA, et al. Megalencephaly and leukodystrophy with mild clinical course: A report on 12 new cases. Brain Dev. 1998;20(3):142-153.

7. De Stefano N, Balestri P, Dotti MT, et al. Severe metabolic abnormalities in the white matter of patients with vacuolating megalencephalic leukoencephalopathy with subcortical cysts. A proton MR spectroscopic imaging study. J Neurol. 2001;248:403-409.

8. Marks HG, Caro PA, Wang ZY, et al. Use of computed tomography, magnetic resonance imaging, and localized $1 \mathrm{H}$ magnetic resonance spectroscopy in Canavan's disease: A case report. Ann Neurol. 1991;30(1):106-110.

9. van der Knaap MS, Naidu S, Breiter SN, et al. Alexander disease: Diagnosis with MR imaging. AJNR Am J Neuroradiol. 2001;22:541-552.

10. Chen CY, Zimmerman RA, Lee CC, et al. Neuroimaging findings in late infantile GM1 gangliosidosis. AJNR Am J Neuroradiol. 1998;19(9):16281630.

11. van der Knaap MS, Smit LM, Barth PG, et al. Magnetic resonance imaging in classification of congenital muscular dystrophies with brain abnormalities. Ann Neurol. 1997;42:50-59. 